\title{
From bladder to systemic syndrome: concept and treatment evolution of interstitial cystitis
}

International Journal of Women's Health

23 July 2015

Number of times this article has been viewed

\author{
Sara Dinis ${ }^{1,2}$ \\ Joana Tavares de Oliveira ${ }^{3,4}$ \\ Rui Pinto ${ }^{1,5}$ \\ Francisco Cruz ${ }^{1,5}$ \\ CA Tony Buffington ${ }^{6}$ \\ Paulo Dinis ${ }^{1,5}$ \\ 'Faculty of Medicine, University \\ of Porto, ${ }^{2}$ Department of Obstetrics \\ and Gynecology, Hospital de São \\ João, Porto, ${ }^{3}$ Faculty of Veterinary \\ Medicine, ULHT, Lisbon, ${ }^{4}$ Institute \\ of Molecular Pathology and \\ Immunology (IPATIMUP), University \\ of Porto, ${ }^{5}$ Department of Urology, \\ Hospital de São João, Porto, Portugal; \\ ${ }^{6}$ Department of Veterinary Clinical \\ Sciences, College of Veterinary \\ Medicine, The Ohio State University, \\ OH, USA
}

\begin{abstract}
Interstitial cystitis, presently known as bladder pain syndrome, has been recognized for over a century but is still far from being understood. Its etiology is unknown and the syndrome probably harbors different diseases. Autoimmune dysfunction, urothelial leakage, infection, central and peripheral nervous system dysfunction, genetic disease, childhood trauma/abuse, and subsequent stress response system dysregulation might be implicated. Management is slowly evolving from a solo act by the end-organ specialist to a team approach based on new typing and phenotyping of the disease. However, oral and invasive treatments are still largely aimed at the bladder and are based on currently proposed pathophysiologic mechanisms. Future research will better define the disease, permitting individualization of treatment.
\end{abstract}

Keywords: bladder pain syndrome, concept, treatment

\section{General concepts Defining bladder pain syndrome/interstitial cystitis, histological classifications}

The European Society for the Study of Interstitial Cystitis (ESSIC) defines bladder pain syndrome (BPS) as chronic (over 6 months) pelvic pain, pressure, or discomfort perceived to be related to the urinary bladder, accompanied by at least one other urinary symptom such as persistent urge to void or frequency in the absence of an identifiable cause. ${ }^{1}$ Although not a life-threatening disease, it is a condition that is a massive burden to the patient. This may easily be underestimated, as often the diagnosis relies solely on the patient's symptoms. These may be so severe that some patients suffering from BPS/interstitial cystitis (IC) present quality-of-life scores lower than those treated with hemodialysis for end-stage renal disease. ${ }^{2}$ Despite this, given the lack of clear knowledge of its etiology, proper management of patients with BPS is still far from fully realized.

Many have tried to define and classify this condition. Nowadays, BPS encompasses a constellation of clinical scenarios, and a histological classification is useful to better characterize the different forms of the disease.

In 1836, Joseph Parrish described a syndrome of chronic frequency, urgency, dysuria, and pelvic pain, which he called "tic doloureux of the bladder."

"Classic" IC refers to the characteristic lesions, initially called Hunner's ulcers, which patients present with. Hunner described what he saw as ulcers using rudimentary nineteenth century cystoscopes. ${ }^{4}$ In fact, Hunner's ulcers are not ulcers but distinct inflammatory lesions that rupture across the mucosa and submucosa upon hydrodistention during cystoscopy. Therefore, they are now referred to as Hunner's lesions. These are observed predominantly in the bladder lateral walls in up to $50 \%$ of the patients. ${ }^{1}$
Correspondence: Paulo Dinis Department of Urology, Hospital São João, 4200 Porto, Portugal

Email paulodinisoliveira@gmail.com 
More frequently observed lesions are glomerulations and petequiae, which are consequences of a friable urothelium; nevertheless, these, like the former, are not pathognomonic. ${ }^{1}$

Because many patients who experience symptoms of BPS do not present intravesical lesions (while some patients without symptoms of BPS do), ${ }^{5}$ and in some cases, there is no documentable inflammation at all, the so-called classic IC is now considered to be part of a wider disorder, presently called the BPS. Thus, this syndrome presents mainly two forms according to histological features: the "classic" form, which presents with identifiable bladder lesions and/or chronic inflammation signs, observed at cystoscopy and histological exam of bladder wall biopsy; and the "non-classic" form in which the symptoms are perceived but there are no macroscopic or even histological changes. A more thorough classification has been proposed by ESSIC, which correlates different possible findings of both cystoscopy and biopsy. ${ }^{6}$

A definition that is both precise and widely accepted is crucial as it allows a more accurate understanding of which treatments are effective in a patient.

\section{Epidemiology}

Although both women and men are affected at any age, the majority of the patients are women. Recent reports, however, suggest that the sex difference in humans may not be as large as originally thought, ${ }^{7,8}$ if it exists at all., ${ }^{9}$

BPS/IC prevalence reports vary depending on the applied diagnostic criteria and the studied population. It has been reported to be as low as $0.06 \%$, although it is thought to be higher. ${ }^{6}$

\section{Etiology/pathophysiology}

Genetic predisposition, autoimmune mechanisms, infection, central neurogenic mechanisms, defective glycosaminoglicanlayer constitution, toxic urinary components, and psychosomatic mechanisms are all candidate players in the pathophysiology of BPS/IC.

\section{Genetic predisposition}

A higher prevalence of BPS/IC among first-degree relatives suggests a genetic (or epigenetic) predisposition. ${ }^{11}$ Furthermore, a recent study found an association among first- and second-degree relatives for fibromyalgia, constipation, and BPS/IC, suggesting that they may have the same underlying genetic factors. ${ }^{12}$ Genetic risk factors for the development of IC including both chromosomal loci and specific genes have been found. ${ }^{13}$ Regarding epigenetics, BPS-specific miRNAs have also been described. ${ }^{14}$

\section{Autoimmune mechanisms}

Diseases known to be caused by autoimmune mechanisms, such as allergies, rheumatoid arthritis, and inflammatory bowel disease, were significantly more frequent among BPS/IC patients. However, any common specific mechanism(s) underlying these associations is yet to be found. ${ }^{15-17}$ BPS type $3 \mathrm{C} /$ classic IC seems to be a distinct inflammatory disease and in many aspects shares features of inflammatory autoimmune diseases. ${ }^{18}$ In an experimental autoimmune cystitis model, chronic pelvic allodynia is mediated by chemokine (C-C motif) ligand 2 (CCL2) through mast cells. ${ }^{19}$ Moreover, uroplakin peptide-specific autoimmunity was shown to initiate BPS/IC-like bladder histology in mice. ${ }^{20}$

\section{Infection}

Evidence of the absence of a current infection should not lead one to assume that an infectious agent does not play a role in the pathogenesis of BPS/IC. Both infectious agents not identified by routine workup, such as nanobacteria, and those that are responsible for a painful response that remains long after the infection has been eradicated, such as an uropathogenic Escherichia coli, are plausible candidates in the disease etiopathogenesis. ${ }^{21}$

Furthermore, although not suggested to colonize the bladder directly, Helicobacter pylori-induced endoluminal damage was recently hypothetized to play a causal role in BPS/IC as well as in atherosclerosis. ${ }^{22}$ In line with such theory, BPS/IC has been reported to increase the risk of coronary heart disease. ${ }^{23}$

\section{Defective glycosaminoglycan-layer constitution}

BPS/IC patients do not necessarily have a defective glycosaminoglycan (GAG)-layer constitution to begin with; instead, they most probably have impaired reparative mechanisms. Nevertheless, preemptive application of a GAG analog, GM-0111, provided varying degrees of protection against bladder insult in a mice model, suggesting that GAG molecules might prevent the development of cystitis by blocking apoptosis and the concurrent release of adenosine triphosphate (ATP) from the urothelium. ${ }^{24}$

Antiproliferative factor is a frizzled protein 8-related sialoglycopeptide identified in approximately $90 \%$ of BPS/IC patients. It delays bladder wall regeneration by inhibiting bladder urothelial cell proliferation. ${ }^{25}$ A disruption of the GAG layer on the outer surface of the urothelium contributes to leakage of irritating substances through the bladder wall. ${ }^{26}$ 
This phenomenon, in turn, might induce inflammatory infiltration with mast cell predominance and increased activation $-70 \%$ compared to $10 \%$ in healthy individuals - and nerve fibers activation. Alternatively, descending central nervous system modulation of peripheral nerves might induce mast cell infiltration with subsequent damage to the urothelium. ${ }^{8}$ Regardless of the underlying mechanisms, decreased expression of tight junction proteins, zonula occludens-1, and occludin with interruption of the bladder urothelium are observed in patients with BPS/IC. ${ }^{27}$

\section{Neurogenic mechanisms}

Both peripheral and central neural sensitization and neuroplasticity have been described in BPS/IC patients, potentially as a consequence of type $\mathrm{C}$ sensory nerve fiber activation. Interestingly, cross-sensitization found in an experimental model of colitis pointed to an overlap between IC and irritable bowel syndrome. ${ }^{28}$

\section{Psychosomatic mechanisms}

Adverse early life events commonly linked to chronic pain disorders, such as child abuse and other adverse childhood experiences, are highly frequent in these patients. $\mathrm{BPS} / \mathrm{IC}$ has been recently described as a functional somatic syndrome. ${ }^{29}$ Stress response system (SRS) alterations are common in humans as well as in cats with a similar disorder called feline IC. While, to our knowledge, no definite genetic predisposition has yet been identified in either cats or humans with this syndrome, findings in cats are at least consistent with the results of some developmental "accident". It has recently been found that when a pregnant female is exposed to a sufficiently harsh stressor, the hormonal products of the ensuing stress response can cross the placenta and affect the course of fetal development, resulting in persistent adrenal dysfunction and sensitization of the central SRS. ${ }^{30}$

\section{BPS/IC as part of a systemic disease}

As earlier described, some of the pathophysiologic mechanisms currently implicated in BPS/IC are also associated with other poorly understood conditions such as inflammatory bowel disease, irritable bowel syndrome, fybromyalgia, or vulvodynia, which in turn frequently coexist with BPS/IC.

The association of BPS/IC with other pain syndromes in patients leads to the realization that it might not be an isolated condition but rather part of a disease with systemic, regional, or organ manifestations varying with each patient.
Other frequently associated non-bladder diseases (NBDs) are depression, panic disorder, migraine, sicca syndrome, allergy, asthma, and temporomandibular joint disorder. The risk of BPS correlates with the number of NBDs. ${ }^{31}$ Also, nonulcer BPS patients present with significantly more depression, panic disorder, migraine, and temporomandibular joint disorder than classic BPS patients. ${ }^{32}$

Symptoms seem to be worse when BPS is associated with systemic pain syndromes such as fibromyalgia. ${ }^{33}$

\section{Diagnostic approach}

In the presence of a suggestive clinical history, confusable diseases (malignancies, infections, neurologic disease, bladder outlet obstruction, and others) must be excluded. After this, patients with symptoms according to the definition should be diagnosed with BPS by the subtype and phenotype. Assessment should include urine analysis, uroflowmetry, cystoscopy with hydrodistension, and bladder biopsy as necessary. ${ }^{1,34}$ A micturition diary and pelvic floor testing are also desirable. A validated symptom and quality-of-life scoring instrument should be considered for initial assessment as well as for follow-up. ${ }^{35}$ Chronic pelvic pain symptoms and quality of life are worse in women than in men. ${ }^{36}$ BPS-associated negative cognitive, behavioral, sexual, or emotional consequences should be assessed. ${ }^{37,38}$ BPS-associated NBDs should be assessed systematically, namely, allergies, other pain syndromes, and psychiatric disorders. ${ }^{39}$ A multidisciplinary approach showed increased severity of symptoms in the presence of nonurologicalassociated somatic syndromes. ${ }^{40}$

Antiproliferative factor, hemoglobin-binding endothelial growth factor, endothelial growth factor, nerve growth factor (NGF), and brain-derived nerve growth factor (BDNF) have all been proposed as biomarkers likely determinable in urine samples. ${ }^{41}$ However, NGF and BDNF also appear to be the most promising biomarkers in overactive bladder. ${ }^{42}$ Even so, NGF may still be of interest to monitor the response to treatment. ${ }^{43}$ Recent application of microarray technology to BPS/IC patients and animal models has given further thrust to this aspect. ${ }^{44}$ The potential utility of infrared microspectroscopy to diagnose BPS/IC has been suggested by the differences found in the concentration of tryptophan and its metabolites in the sera. ${ }^{45,46}$ On a related subject, mid-infrared microspectroscopy and metabolomic analysis identified changes in the tryptophan catabolism pathway that differentiated patients with fibromyalgia from those with osteoarthritis and rheumatoid arthritis. ${ }^{47}$ Nuclear magnetic resonance (NMR)-based global metabolomics revealed a 
strong signature of BPS/IC. Among other signals that were higher in the BPS/IC group, tyramine, the pain-related neuromodulator, and 2-oxoglutarate were significantly elevated in the urine specimens of BPS/IC patients. ${ }^{48}$ Contrast-enhanced magnetic resonance imaging (CE-MRI) has recently been shown to be of potential interest as a clinical diagnostic method for BPS/IC by monitoring the loss of permeability of the primary bladder urothelium. ${ }^{49}$

The recent criteria of BPS/IC described by ESSIC are far wider than the former National Institute of Diabetes and Digestive and Kidney Diseases criteria. As the number of patients diagnosed with this disease increased, so did the presenting clinical features. Since patients with ESSIC-defined diagnosis present with different characteristics, it is possible that treatments that were adequate for patients diagnosed earlier do not have the same effects as in National Institute of Diabetes and Digestive and Kidney Diseases-defined patients. Patel and Evans suggested a treatment algorithm in which the management is based on the patient phenotype that divides the patient's symptoms into six categories: urinary, psychological, organ-specific, infection, neurologic, and tenderness. ${ }^{50}$ This would allow the comparison between studies as well as tweaking of individual patient treatment.

\section{Management}

Whenever treating a BPS/IC patient, realistic expectations should be kept in mind. Currently, there is no curative treatment, and so the primary goal remains an improvement in the patient's quality of life. Furthermore, even within the available treatment options, valid studies are scarce.

\section{First-line conservative management}

Management options for BPS/IC found in the literature may be conflicting; however, most relevant guidelines propose that treatment should initially be conservative. ${ }^{51}$

When deciding on a conservative option, one should take into consideration patients' complaints and their past experiences. Those patients who can identify certain pain triggers related to food and beverages will benefit from appropriate life changes. The most common triggers are citrate and drinks that contain caffeine or alcohol or are carbonated. Other sources of alternative and complementary medicine, such as dietary supplementation and acupuncture, are currently also considered. ${ }^{52}$ Multidisciplinary approach and help should be envisaged right from the start, in opposition to more current views in which the pelvic pain patient is managed by a single so-called end-organ specialist, to the limit of doctor and patient endurance. Based on urinary, psychological, organ-specific, infection, neurologic, and tenderness or similar comprehensive classifications, all outstanding related complaints should be addressed. At this early phase, associated or concomitant psychological and psychiatric aspects should be sought out and treated. A correct diagnosis and eventual treatment of the personality type, possible childhood trauma/sexual abuse, anxiety disorder, depression, and reaction to pain are crucial for patient well-being. Simple explanation of chronic pain and what to expect from its evolution and treatment, or the mere assurance of a real existing diagnosis is paramount. This cannot be achieved without interspecialty partnership between medical specialties, nursing, psychology, physiotherapists. Pelvic-floor hypertonicity is extremely common in BPS. ${ }^{53}$ It enhances pain and may be a cause by itself. Furthermore, hypertonic pelvic floor muscles will induce dysfunctional voiding with the ensuing plethora of complaints, both micturitional and proctologic, namely, hesitancy, frequency, slow stream, and constipation. ${ }^{54}$ Hence the need for physiotherapy for pelvic floor muscle relaxation should be assessed. Stress-reducing techniques may be helpful. In this first-line approach, over-the-counter analgesics should be used on demand. ${ }^{55}$ NBDs that often coexist with BPS must be diagnosed and treated simultaneously, namely fibromyalgia and chronic fatigue.

\section{Level I evidence treatments Oral treatments}

Standard oral treatment includes amitriptyline, pentosan polysulfate, and hydroxyzine.

van Ophoven and collaborators published a randomized controlled trial (RCT) in 2004 confirming the usefulness of amitriptyline. ${ }^{56}$ Amitriptyline decreases 5-hydroxytriptamine reuptake and also stabilizes mast cells acting as an anticholinergic and sedative. ${ }^{57,58}$ Of note, an RCT by the Interstitial Cystitis Collaborative Research Network showed that only doses $>50 \mathrm{mg} /$ day seem to be effective. ${ }^{59}$ Interestingly, diagnosis by the ESSIC criteria does not seem to decrease the rate of responding patients when treated with amytriptiline. Cystoscopic findings also do not influence patient outcomes. ${ }^{60}$

The US Food and Drug Administration (FDA)-approved pentosan polysulfate sodium (PPS) ${ }^{61}$ replaces the GAG layer and inhibits mast cell degranulation, thus targeting two putatively important physiopathologic mechanisms of BPS/IC. ${ }^{26}$ Administering a concomitant low dose of heparin subcutaneously is more effective than administering PPS alone in patients who initially responded to oral PPS. ${ }^{61}$ Regarding the mechanism of action of oral PPS, in female 
B6C3F1/N mice, its administration stimulated the immune system, increasing the numbers of specific cell populations and enhancing macrophage phagocytosis and natural killer (NK) cell activity. ${ }^{62}$ Recently, however, results from a randomized, double-blind, placebo-controlled study of patients with symptoms consistent with IC did not demonstrate a treatment effect with PPS when compared with placebo at the currently established dose. ${ }^{63}$

Hydroxyzine, a histamine type 1 (H1)-receptor antagonist, blocks histamine release in the bladder by inhibiting mast cell degranulation. Although considered standard oral treatment in major guidelines, ${ }^{51}$ there are no RCTs supporting its use. A pilot study showed $40 \%$ decrease overall and a $55 \%$ decrease in severity of symptoms in a subgroup of patients with personal history of allergies. ${ }^{59,64}$ These results underline the importance of phenotyping the patient when deciding which oral agent might be more effective.

In an RCT, cyclosporine A, an immunosuppressant drug that inhibits $\mathrm{T}$-cell activation and cytokine release, was proven to be significantly more effective when compared with PPS. Nevertheless, cyclosporine A has significantly more serious adverse effects than PPS. ${ }^{65}$ Cyclosporine A was also found to be highly effective for patients with Hunner's lesions refractory to conservative options in a retrospective study. As its considerable number of side effects may justify therapy dropout, these remain a significant drawback while using this seemingly attractive treatment. ${ }^{66}$

\section{Intravesical instillations and injections}

Intravesical treatments with proven efficacy include PPS, dimethyl sulfoxide (DMSO), and onabotulinum toxin A (OnabotA) plus hydrodistension. An RCT showed significantly increased efficacy of simultaneous oral and intravesical PPS use when compared with placebo or oral PPS alone. Intravesically instilled PPS allows higher concentrations to be achieved in the bladder. ${ }^{67}$

FDA-approved DMSO for intravesical use in BPS/IC inhibits mast cells and presents analgesic, anti-inflammatory, muscle relaxant, and collagen-dissolution effects. ${ }^{68}$ In a randomized, controlled study of DMSO compared to placebo, subjective improvement was reported in 53\% and objective improvement was observed in $93 \%$ of patients. ${ }^{69}$ Intravesical DMSO effects were shown to be increased and prolonged with the concomitant use of hyperbaric oxygen therapy. ${ }^{70}$ The initial flare-up in IC/PBS symptoms after DMSO treatment was recently related to the presence of lactate dehydrogenase in the treatment medium, suggesting that DMSO permeabilizes urothelial membranes causing leakage of cytosolic contents including ATP and Ach rather than enhancing the release of these mediators. ${ }^{71}$

An RCT study analyzing the difference between hydrodistension and hydrodistension plus intravesical OnabotA injection showed symptomatic improvement in all groups. However, $70 \%$ of patients in the hydrodistension group had returned to their previous symptoms after the first month, whereas in the OnabotA-treated groups there was still improvement of pain visual analog scale, functional bladder capacity, and cystometric bladder capacity at 3 months. At 12 and 24 months, the results in the active group were 55\% and 30\% versus $26 \%$ and $17 \%$ in the hydrodistension group. ${ }^{72}$

\section{Lower evidence level treatment options Oral treatments}

Oral H2-receptor antagonists are still being used, mainly supported by an RCT that showed significant benefit of cimetidine relative to placebo. ${ }^{73}$ However, no recent publications with this drug group were found.

\section{Intravesical treatments}

More recently, in an open-label uncontrolled study intravesical instillation of pentosan polysulfate encapsulated in a liposome nanocarrier suggested a significant decrease in frequency, urgency, and pain, and improvement in the quality of life for 2 months. ${ }^{74}$

In a study by Pinto et al OnabotA trigonal-only injection without hydrodistension seemed effective and long lasting. An improvement after a 3-month follow-up period was reported by $87 \%$ of patients $(\mathrm{N}=23)$. Moreover, more than $50 \%$ of patients reported continued beneficial effects 9 months after the first treatment. Whenever retreatment was needed, similar results were obtained. Both ulcer- and non-ulcer-bearing patients reported similar clinical benefit. ${ }^{75}$ Another longer lasting prospective trial confirmed that repeated trigonal injection of OnabotA provides consistent improvement on BPS/IC symptoms without significant complications. ${ }^{76}$ Other groups have performed prospective trials with repeated OnabotA injection, further proving it to be safe and effective. ${ }^{77}$ At the cellular level, OnabotA's clinical benefits seem to be associated with decreased markers of chronic inflammation and decreased expression of apoptotic signaling molecules. ${ }^{78}$ Moreover, downregulation of the vascular endothelial growth factor was associated with decreased inflammation after intravesical OnabotA injections combined with hydrodistention in IC patients. ${ }^{79}$ Vascular endothelial growth factor is a target gene of the 
hypoxia-inducible factor-1 $\alpha$ (HIF1alpha) overexpressed in the bladder of BPS/IC patients. ${ }^{80}$

Thirty non-ulcer- and ten ulcer-bearing BPS/IC treatmentrefractory patients received intravesical botulinum toxin $\mathrm{A}$ injection, with clinical improvement being observed only in patients without Hunner's ulcers. ${ }^{81}$ On the other hand, in another study, ulcerative and nonulcerative forms of BPS/IC did not differ in symptom intensity or response to OnabotA. ${ }^{82}$

This further points to the need to better characterize the two subsets of patients and to decide the most suitable treatment accordingly. For chronic refractory BPS/IC patients, although a significant benefit was noted in a small number of patients, in a multicenter, prospective, randomized, doubleblind study to measure treatment effectiveness, abobotulinum toxin A was associated with no overall improvement in total O’Leary Sant (OLS) score. ${ }^{83}$

GAG layer restitution has been attempted with other GAGs, namely, hyaluronic acid and chondroitin sulfate. Small studies have proposed chondroitin sulfate as a form of intravesical treatment with somewhat positive results. ${ }^{59}$ However, two multicentric, placebo-controlled RCTs evaluating intravesical sodium chondroitin sulfate efficacy and safety failed to demonstrate a statistically significant improvement. Therefore, although safe, the benefit of this treatment is minor and it should not be used as a monotherapy. ${ }^{84,85}$

Nevertheless, a meta-analysis revealed a significant improvement in patients' quality of life when combining the results of the three small studies using intravesical chondroitin sulfate. Larger studies are warranted, taking into account the identification of the specific subtype of BPS/IC patient who might profit from chondroitin sulfate treatment. ${ }^{86}$

Regarding hyaluronic acid, some clinical benefit was achieved in BPS/IC patients. A 6-year prospective study involving 126 patients performed to assess the long-term effect of intravesical hyaluronic acid therapy showed overall $87 \%$ positive response to treatment. ${ }^{87}$ Fifty percent of patients remained asymptomatic after the observational period. Recurrence during the first year was reported after initial improvement by $41.7 \%$ patients. Only $8.3 \%$ seemed to be refractory to intravesical hyaluronic acid treatment. It is noteworthy that the patients in this study were a subset showing a positive modified potassium test. ${ }^{87}$ Intravesical hyaluronic acid instillation was an effective and safe treatment for 33 patients with refractory IC/PBS, with no influence of previous treatment modalities and presence of Hunner's ulcer being observed to affect its efficacy. ${ }^{88}$ Along with the reduction of BPS/IC symptoms, intravesical hyaluronic acid also seemed to improved sexual function. ${ }^{89}$
In a recent work by Porru et al 22 patients with BPS/IC received intravesical instillations $(40 \mathrm{~mL})$ of sodium hyaluronic acid $1.6 \%$ and chondroitin sulfate $2.0 \%$ in $0.9 \%$ saline solution once weekly for 8 weeks, and then once every 2 weeks for the next 6 months. The use of intravesical sodium chondroitin sulfate and hyaluronic acid together showed encouraging results both on the mucosa reconstitution and clinical improvement, with significant reductions in urgency and pain scores. ${ }^{90,91}$ Yet, another study comparing intravesical hyaluronic acid and hyaluronic acid/chondroitin sulfate therapy for patients with BPS/IS showed no difference between groups, with statistical improvements observed in both groups at 6 months. ${ }^{92}$

\section{Neuromodulation, bladder training, physical therapy}

Sacral neuromodulation aims to reverse the chronic upregulation of the pelvic nerves believed to be involved in the pathophysiology of BPS/IC. ${ }^{33}$ Gajewski et al's long-term results showed improvement of $72 \%$ of the patients after a median follow-up of 61.5 months. High rates of revision were nevertheless observed, and in $28 \%$ of the patients explantation was necessary. Therefore, neuromodulation should be thought of in persistent cases before advancing to more invasive interventions such as surgery. ${ }^{94,95}$

Some patients present pelvic floor tenderness during physical exam. These are those likely to benefit from physical therapy. After ten sessions, myofascial physical therapy significantly improved the patients' global symptoms. ${ }^{50}$ Fitzgerald et al's randomized, multicenter clinical trial of myofascial physical therapy in women with IC/painful bladder syndrome and pelvic floor tenderness reported a significant 59\% improvement rate in the treatment group versus $26 \%$ in the placebo group..$^{96}$

\section{Surgical treatments}

Whenever Hunner's lesions are present, transurethral resection induces a good response in $90 \%$ of the patients. Transurethral resection combined with hydrodistention is also an effective treatment option. ${ }^{97}$ Transurethral lesion destruction has also been obtained both by fulguration and by laser application with success in an attempt to lessen morbidity. Patients undergoing transurethral intervention remain symptom-free for up to 2 years. Nevertheless, this invasive treatment is effective only in the particular subset of BPS/IC patients with Hunner's lesion (ESSIC type 3C). ${ }^{98,99}$ Electrosurgical management of Hunner's lesions seemed to be an effective and safe procedure without diminishing bladder capacity. ${ }^{100}$ 
Data on surgical treatment are largely variable, and therefore major surgery must be looked at as a last resort. ${ }^{101}$ Careful patient selection is of major importance, and available evidence identifies end-stage classic ulcerative disease with bladder contraction as the ideal indication for surgery. Urinary diversion with bladder conservation, supratrigonal cystectomy, subtrigonal cystectomy, and radical cystectomy, including excision of the urethra, have all been described. Supratrigonal cystectomy with enterocystoplasty is the most well-accepted one. ${ }^{102,103}$

\section{Perspectives}

A randomized, double-blind, placebo-controlled, low-dose sildenafil trial for IC showed a significant improvement in the symptom, problem index scores, and urodynamic index in the treatment group, with the efficiency of treatment reaching $62.5 \%$. Significant changes of the VAS values were observed by week $12 .{ }^{104}$

Mouse models suggest that modulation of the cannabinoid $\mathrm{CB} 2$ receptors might be a promising therapeutic strategy for IC. ${ }^{105,106}$

Herpes simplex virus vector-mediated encoding human preproenkephalin, the precursor of met- and leukoenkephalin (which are endogenous opioids acting on $\delta$-opioid receptors), has demonstrated physiological improvement in visceral pain induced by bladder irritation, thus may be a potentially useful treatment modality for BPS/IC. ${ }^{107}$ Recent work reports on the relatively large magnitude of placebo-associated responses in IC patients. ${ }^{108,109}$ One can envision at least two reasons that make it all the more pertinent: 1) Non-placebo-controlled trials are of limited usefulness for evaluation of therapies for BPS/IC. It has been known since the early DMSO studies, which found that water was approximately $50 \%$ effective. Recently, in a randomized, double-blind, placebo-controlled trial of adalimumab for IC/bladder, although the treatment resulted in a statistically significant improvement in outcome measures compared to baseline in patients with moderate to severe BPS/IC, it failed to demonstrate positive proof of concept compared to placebo due to a significant placebo effect. ${ }^{108}$ 2) Benedetti points to the therapeutic relationship between the caregiver and the patient as being very important to patient outcome. ${ }^{110}$ In fact, data on spontaneously occurring IC animal models strongly suggest that this may be considered a "new line of therapy" to be investigated. ${ }^{111}$

Differences in sympathetic nervous system function have been identified in cats and humans, as well as in adrenocorticotropic hormone and cortisol levels. ${ }^{112,113}$ Charrua et al found elevated levels of noradrenaline in samples of both blood and urine of BPS/IC patients who also presented with the altered Tilt Test, indicating sympathetic dysfunction. In the same work, treatment with high doses of adrenaline induced both clinical and histological features akin to BPS/IC in the bladder and nervous system of Wistar rats. ${ }^{114}$ In animal models, both in utero and early life, stressors can induce SRS alteration, resulting in enhanced adrenocorticotropic hormone production and low cortisol, along with enhanced sympathetic system activity. ${ }^{30}$ These alterations, also observed in humans with posttraumatic stress syndrome, are the result of epigenetic changes possibly imprinted in a genetically susceptible individual. ${ }^{115}$ In this regard, future treatment will certainly be aimed at reversing those epigenetic alterations. This might be achieved with pharmacological and nonpharmacological (behavioral) treatment. ${ }^{116}$ Furthermore, observations about the so-called context (including placebo) effect and the underlying biochemical basis for clinical improvement bring to light the necessity of careful counseling and close psychological and multidisciplinary support, preferably from the start of patient management.

\section{Conclusion}

Treatment of BPS/IC remains a clinical challenge. Besides basic diagnosis, typing and phenotyping of the disease is paramount to any measure of success. Management should start with adequate counseling and conservative measures. Oral medication is proven useful despite the probable heterogeneity of BPS/IC. However, for Hunner's lesion and nonresponding other types of the disease, surgery is indicated. Non-bladder conditions should be sought out and treated simultaneously with bladder complaints. Further research on the etiology of the disease is paramount to curative treatment(s).

\section{Disclosure}

Professor Cruz has received honoraria, grants and travel expenses from Astellas and Allergan. The remaining authors declare no conflicts of interest in this work.

\section{References}

1. van de Merwe JP, Nordling J, Bouchelouche P, et al. Diagnostic criteria, classification, and nomenclature for painful bladder syndrome/interstitial cystitis: an ESSIC proposal. Eur Urol. 2008;53:60-67.

2. Chancellor MBA. Multidisciplinary consensus meeting on IC/PBS: outcome of the consensus meeting on interstitial cystitis/painful bladder syndrome. Rev Urol. 2007;9:81-83.

3. Parsons JK, Parsons CL. The historical origins of interstitial cystitis. J Urol. 2004;171:20-22.

4. Hunner GL. A rare type of bladder ulcer in women: report of cases. Boston Med Soc J. 1915;172:247-292.

5. Waxman JA, Sulak PJ, Kuehl TJ. Cystoscopic findings consistent with interstitial cystitis in normal women undergoing tubal ligation. J Urol. 1998;160:1663-1667. 
6. Engeler D, Baranowski AP, Borovicka J, et al. EAU Guidelines on Chronic Pelvic Pain. Uroweb; 2014.

7. Clemens JQ, Meenan RT, Rosetti MC, Gao SY, Calhoun EA. Prevalence and incidence of interstitial cystitis in a managed care population. J Urol. 2005;173:98-102.

8. Jones CA, Nyberg L. Epidemiology of interstitial cystitis. Urology. 1997;49:2-9.

9. Miller JL, Rothman I, Bavendam TG, Berger RE. Prostatodynia and interstitial cystitis: one and the same? Urology. 1994;45:587-590.

10. Suskind AM, Berry SH, Ewing BA, Elliott MN, Suttorp MJ, Clemens JQ. The prevalence and overlap of interstitial cystitis/bladder pain syndrome and chronic prostatitis/chronic pelvic pain syndrome in men: results of the RAND interstitial cystitis epidemiology male study. J Urol. 2013; 189:141-145.

11. Warren JW, Jackson TL, Langenberg P, Meyers DJ, Xu J. Prevalence of interstitial cystitis in first-degree relatives of patients with interstitial cystitis. Urology. 2004;63:17-21.

12. Allen-Brady K, Norton PA, Cannon-Albright L. Risk of associated conditions in relatives of subjects with interstitial cystitis. Female Pelvic Med Reconstr Surg. 2014;21(2):93-98.

13. Domschke K, Maron E. Genetic factors in anxiety disorders. Mod Trends Pharmacopsychiatri. 2013;29:24-46.

14. Gheinani AH, Burkhard FC, Monastyrskaya K. Deciphering microRNA code in pain and inflammation: lessons from bladder pain syndrome. Cell Mol Life Sci. 2013;70(20):3773-3789.

15. Chang FY, Lu CL. Irritable bowel syndrome and migraine: bystanders or partners? J Neurogastroenterol Motil. 2013;19(3):301-311.

16. Martínez-Martínez LA, Mora T, Vargas A, Fuentes-Iniestra M, Martínez-Lavín M. Sympathetic nervous system dysfunction in fibromyalgia, chronic fatigue syndrome, irritable bowel syndrome, and interstitial cystitis: a review of case-control studies. J Clin Rheumatol. 2014;20(3):146-150.

17. Smorgick N, Marsh CA, As-Sanie S, Smith YR, Quint EH. Prevalence of pain syndromes, mood conditions, and asthma in adolescents and young women with endometriosis. J Pediatr Adolesc Gynecol. 2013; 26(3):171-175.

18. Logadottir Y, Delbro D, Lindholm C, Fall M, Peeker R. Inflammation characteristics in bladder pain syndrome ESSIC type 3C/classic interstitial cystitis. Int J Urol. 2014;21(suppl 1):75-78.

19. Bicer F, Altuntas CZ, Izgi K, et al. Chronic pelvic allodynia is mediated by CCL2 through mast cells in an experimental autoimmune cystitis model. Am J Physiol Renal Physiol. 2014;10:ajrenal.00202.

20. Izgi K, Altuntas CZ, Bicer F, et al. Uroplakin peptide-specific autoimmunity initiates interstitial cystitis/painful bladder syndrome in mice. PLoS One. 2013;8(8):e72067.

21. Rosen JM, Klumpp DJ. Mechanisms of pain from urinary tract infection. Int J Urol. 2014;21(suppl 1):26-32.

22. Verit A, Güner ND. Helicobacter pylori and urinary system stones: endoluminal damage as sub-hypothesis to support the current stone theory. Med Hypotheses. 2014;83(6):677-680.

23. Chen HM, Lin CC, Kang CS, Lee CT, Lin HC, Chung SD. Bladder pain syndrome/interstitial cystitis increase the risk of coronary heart disease. Neurourol Urodyn. 2014;33(5):511-515.

24. Lee WY, Savage JR, Zhang J, Jia W, Oottamasathien S, Prestwich GD. Prevention of anti-microbial peptide LL-37-induced apoptosis and ATP release in the urinary bladder by a modified glycosaminoglycan. PLoS One. 2013;8(10):e77854.

25. Keay S. Cell signaling in interstitial cystitis/painful bladder syndrome. Cell Signal. 2008;20:2174-2179.

26. Teichman JM, Moldwin R. The role of the bladder surface in interstitial cystitis/painful bladder syndrome. Can J Urol. 2007;14(4): 3599-3607.

27. Lee JD, Lee MH. Decreased expression of zonula occludens-1 and occludin in the bladder urothelium of patients with interstitial cystitis/ painful bladder syndrome. J Formos Med Assoc. 2014;113(1): $17-22$.
28. Yoshikawa S, Kawamorita N, Oguchi T, et al. Pelvic organ crosssensitization to enhance bladder and urethral pain behaviors in rats with experimental colitis. Neuroscience. 2015;284:422-429.

29. Warren JW. Bladder pain syndrome/interstitial cystitis as a functional somatic syndrome. J Psychosom Res. 2014;77(6):510-515.

30. Buffington CAT. Comorbidity of interstitial cystitis with other unexplained clinical conditions. J Urol. 2004;172:1242-1248.

31. Warren JW, Wesselmann U, Morozov V, Langenberg PW. Numbers and types of nonbladder syndromes as risk factors for interstitial cystitis/ painful bladder syndrome. Urology. 2011;77(2):313-319.

32. Peters KM, Killinger KA, Mounayer MH, Boura JA. Are ulcerative and nonulcerative interstitial cystitis/painful bladder syndrome 2 distinct diseases? A study of coexisting conditions. Urology. 2011; 78(2):301-308.

33. Nickel JC, Tripp DA, Pontari M, et al. Interstitial cystitis/painful bladder syndrome and associated medical conditions with an emphasis on irritable bowel syndrome, fibromyalgia and chronic fatigue syndrome. J Urol. 2010;184(4):1358-1363.

34. Parsons CL. Diagnosing the bladder as the source of pelvic pain: successful treatment for adults and children. Pain Manag. 2014; 4(4):293-301.

35. Marsha KG, Toby CC. Interstitial cystitis/painful bladder syndrome (IC/PBS): is there anything new under the sun? Curr Obstet Gynecol Rep. 2014;3:165-171.

36. Clemens JQ, Clauw DJ, Kreder K, et al; MAPP Research Network. Comparison of baseline urologic symptoms in men and women in the multidisciplinary approach to the study of chronic pelvic pain (Mapp) research cohort. J Urol. 2014;193(5):1554-1558.

37. Liu B, Su M, Zhan H, Yang F, Li W, Zhou X. Adding a sexual dysfunction domain to upoint system improves association with symptoms in women with interstitial cystitis and bladder pain syndrome. Urology. 2014;84(6):1308-1313.

38. Quaghebeur J, Wyndaele JJ. Prevalence of lower urinary tract symptoms and level of quality of life in men and women with chronic pelvic pain. Scand J Urol. 2014;2:1-8.

39. Nickel JC, Irvine-Bird K, Jianbo L, Shoskes DA. Phenotype-directed management of interstitial cystitis/bladder pain syndrome. Urology. 2014;84(1):175-179.

40. Krieger JN, Stephens AJ, Landis JR, et al; MAPP Research Network. Relationship between chronic non-urological associated somatic syndromes (NUAS) and symptom severity in urological chronic pelvic pain syndromes: baseline evaluation of the multidisciplinary approach to the study of chronic pelvic pain (MAPP) study. J Urol. 2015;193(4):1254-1262.

41. Qu HC, Zhang W, Yan S, Liu YL, Wang P. Urinary nerve growth factor could be a biomarker for interstitial cystitis/painful bladder syndrome: a meta-analysis. PLoS One. 2014;9(9):e106321.

42. Bhide AA, Cartwright R, Khullar V, Digesu GA. Biomarkers in overactive bladder. Int Urogynecol J. 2013;24(7):1065-1072.

43. Jiang YH, Liu HT, Kuo HC. Decrease of urinary nerve growth factor but not brain-derived neurotrophic factor in patients with interstitial cystitis/bladder pain syndrome treated with hyaluronic acid. PLoS One. 2014;9(3):e91609.

44. Yang W, Kim Y, Kim TK, et al. Integration analysis of quantitative proteomics and transcriptomics data identifies potential targets of frizzled-8 protein-related antiproliferative factor in vivo. BJU Int. 2012;110 (11 pt C):E1138-E1146.

45. Rubio-Diaz DE, Pozza ME, Dimitrakov J, et al. A candidate serum biomarker for bladder pain syndrome/interstitial cystitis. Analyst. 2009;134(6):1133-1137.

46. Hsieh TF, Yu KJ, Lin SY. Possible application of Raman microspectroscopy to verify the interstitial cystitis diagnosis after potassium sensitivity test: phenylalanine or tryptophan as a biomarker. Dis Markers. 2007;23(3):147-152.

47. Hackshaw KV, Rodriguez-Saona L, Plans M, Bell LN, Buffington CA. A bloodspot-based diagnostic test for fibromyalgia syndrome and related disorders. Analyst. 2013;138(16):4453-4462. 
48. Wen H, Lee T, You S, et al. Urinary metabolite profiling combined with computational analysis predicts interstitial cystitis-associated candidate biomarkers. J Proteome Res. 2014;14(1):541-548.

49. Towner RA, Smith N, Saunders D, et al. Contrast-enhanced magnetic resonance imaging (CE-MRI) as a diagnostic tool for assessing bladder permeability and associated colon cross-talk: pre-clinical studies in a rat model. J Urol. 2014;193(4):1394-1400.

50. Patel BN, Evans RJ. Overactive bladder and pain: management strategies. Curr Urol Rep. 2010;11(6):379-384.

51. Hanno PM, Burks DA, Quentin Clemens' J, et al. Diagnosis and Treatment of Interstitial Cystitis/Bladder pain Syndrome. American Urological Association (AUA) Guideline; 2014.

52. Leong FC. Complementary and alternative medications for chronic pelvic pain. Curr Pain Headache Rep. 2011;15(5):343-346.

53. Hoffman D. Understanding multisymptom presentations in chronic pelvic pain: the inter-relationships between the viscera and myofascial pelvic floor dysfunction. Obstet Gynecol Clin North Am. 2014; 41(3):503-510.

54. Hartmann D, Sarton J. Chronic pelvic floor dysfunction. Best Pract Res Clin Obstet Gynaecol. 2014;28(7):977-990.

55. Pergialiotis V, Arnos P, Mavros MN, PitsouniE, Athanasiou S, Falagas ME. Urinary tract analgesics for the treatment of patients with acute cystitis: where is the clinical evidence? Expert Rev Anti Infect Ther. 2012; 10(8):875-879.

56. van Ophoven A, Pokupic S, Heinecke A, Hertle L. A prospective, randomized, placebo controlled, double-blind study of amitriptyline for the treatment of interstitial cystitis. J Urol. 2004;172:533-536.

57. Marinkovic SP, Moldwin R, Gillen LM, Stanton SL. The management of interstitial cystitis or painful bladder syndrome in women. BMJ. 2009;339:b2707.

58. Foster HE Jr, Hanno PM, Nickel JC, et al. Interstitial cystitis collaborative research network. Effect of amitriptyline on symptoms in treatment naïve patients with- interstitial cystitis/painful bladder syndrome. JUrol. 2010; 183:1853-1858.

59. Fall M, Oberpenning F, Peeker R. Treatment of bladder pain syndrome/ interstitial cystitis 2008: can we make evidence-based decisions? Eur Urol. 2008;54:65-75.

60. Sun Y, Fang Z, Ding Q, Zheng J. Effect of amitriptyline in treatment interstitial cystitis or bladder pain syndrome according to two criteria: does ESSIC criteria change the response rate? Neurourol Urodyn. 2014;33(3):341-344

61. Moutzouris DA, Falagas ME. Interstitial cystitis: an unsolved enigma. Clin J Am Soc Nephrol. 2009;4:1844-1857.

62. Thakur SA, Nyska A, White KLJr, SmithMJ, Auttachoat W, Germolec DR. Immunomodulatory activity of orphan drug Elmiron ${ }^{\circledR}$ in female B6C3F1/N mice. Food Chem Toxicol. 2014;68:196-203.

63. Nickel JC, Herschorn S, Whitmore KE, et al. Pentosan polysulfate sodium for treatment of interstitial cystitis-bladder pain syndrome: insights from a randomized, double-blind, placebo controlled study. J Urol. 2014;193(3):857-862.

64. Theoharides TC, Sant GR. Hydroxyzine therapy for interstitial cystitis. Urology. 1997;49(5A suppl):108-110.

65. Sairanen J, Tammela TL, Leppilahti M, et al. Cyclosporine A and pentosanpolysulfate sodium for the treatment of interstitial cystitis: a randomized comparative study. $J$ Urol. 2005;174:2235-2238.

66. Forrest JB, Payne CK, Erickson DR. Cyclosporine A for refractory interstitial cystitis/bladder pain syndrome: experience of 3 tertiary centers. J Urol. 2012;188:1186-1191.

67. Davis EL, El Khoudary SR, Talbott EO, Davis J, Regan LJ. Safety and efficacy of the use of intravesical and oral pentosanpolysulfate sodium for interstitial cystitis: a randomized double-blind clinical trial. J Urol. 2008;179:177-185.

68. Nordling J. Interstitial cystitis: how should we diagnose it and treat it in 2004? Curr Opin Urol. 2004;14:323-327.

69. Perez-Marrero R, Emerson LE, Feltis JT. A controlled study of dimethyl sulfoxide in interstitial cystitis. J Urol. 1988;140:36-39.
70. Gallego-Vilar D, García-Fadrique G, Povo-Martin I, Salvador-Marin M, Gallego-Gomez J. Maintenance of the response to dimethyl sulfoxide treatment using hyperbaric oxygen in interstitial cystitis/painful bladder syndrome: a prospective, randomized, comparative study. Urol Int. 2013;90:411-416.

71. Smith KJ, Chess-Williams R, McDermott C. Luminal DMSO: effects on detrusor and urothelial/lamina propria function. Biomed Res Int. 2014;2014:347616.

72. Kuo HC, Chancellor MB. Comparison of intravesicalbotulinum toxin type A injections plus hydrodistention with hydrodistention alone for the treatment of refractory interstitial cystitis/painful bladder syndrome. BJU Int. 2009;104:657-661.

73. Thilagarajah R, Witherow RO, Walker MM. Oral cimetidine gives effective symptom relief in painful bladder disease: a prospective, randomized, double-blind placebo-controlled trial. BJU Int. 2001; $87: 207-212$

74. Lander EB, See JR. Intravesical instillation of pentosan polysulfate encapsulated in a liposome nanocarrier for interstitial cystitis. Am J Clin Exp Urol. 2014;2(2):145-148.

75. Pinto R, Lopes T, Frias B, et al. Trigonal injection of botulinum toxin A in patients with refractory bladder pain syndrome/interstitial cystitis. Eur Urol. 2010;58:360-365.

76. Pinto R, Lopes T, Silva J, Silva C, Dinis P, Cruz F. Persistent therapeutic effect of repeated injections of onabotulinum toxin a in refractory bladder pain syndrome/interstitial cystitis. J Urol. 2013;189:548-553.

77. Kuo HC. Repeated intravesicalona botulinum toxin A injections are effective in treatment of refractory interstitial cystitis/bladder pain syndrome. Int J Clin Pract. 2013;67:427-434.

78. Shie JH, Liu HT, Wang YS, Kuo HC. Immunohistochemical evidence suggests repeated intravesical application of botulinum toxin A injections improve treatment efficacy of interstitial cystitis/bladder pain syndrome. BJU Int. 2013;111:638-646.

79. Peng $\mathrm{CH}$, Jhang JF, Shie JH, Kuo HC. Down regulation of vascular endothelial growth factor is associated with decreased inflammation after intravesical OnabotulinumtoxinA injections combined with hydrodistention for patients with interstitial cystitis - clinical results and immunohistochemistry analysis. Urology. 2013;82(6):e1-e6.

80. Lee JD, Lee MH. Metallothionein overexpression of bladder biopsies associated with tissue hypoxia in patients with interstitial cystitis/painful bladder syndrome. Int J Urol. 2014;21(7):719-723.

81. Lee CL, Kuo HC. Intravesicalbotulinum toxin a injections do not benefit patients with ulcer type interstitial cystitis. Pain Physician. 2013;16:109-116.

82. Pinto R, Lopes T, Costa D, et al. Ulcerative and nonulcerative forms of bladder pain syndrome/interstitial cystitis do not differ in symptom intensity or response to onabotulinum toxin A. Urology. 2014; 83(5):1030-1034.

83. Manning J, Dwyer P, Rosamilia A, Colyvas K, Murray C, Fitzgerald E. A multicentre, prospective, randomised, double-blind study to measure the treatment effectiveness of abobotulinum A (AboBTXA) among women with refractory interstitial cystitis/bladder pain syndrome. Int Urogynecol J. 2014;25(5):593-599.

84. Nickel JC, Egerdie RB, Steinhoff G, Palmer B, Hanno P. A multicenter, randomized, double-blind, parallel group pilot evaluation of the efficacy and safety of intravesical sodium chondroitin sulfate versus vehicle control in patients with interstitial cystitis/painful bladder syndrome. Urology. 2010;76:804-809.

85. Nickel JC, Hanno P, Kumar K, Thomas H. Second multicenter, randomized, double-blind, parallel group evaluation of the effectiveness and safety of intravesical sodium chondroitin sulfate compared with inactive vehicle control in subjects with interstitial cystitis/painful bladder syndrome. Urology. 2012;79:1224-1225.

86. Thakkinstian A, Nickel JC. Efficacy of intravesical chondroitin sulphate in treatment of interstitial cystitis/bladder pain syndrome (IC/BPS/IC): individual patient data (IPD) meta-analytical approach. Can Urol Assoc J. 2013;7:195-200. 
87. Engelhardt PF, Morakis N, Daha LK, Esterbauer B, Riedl CR. Long-term results of intravesical hyaluronan therapy in bladder pain syndrome/interstitialcystitis. Int Urogynecol J Pelvic Floor Dysfunct. 2011;22:401-405.

88. Kim A, Lim B, Song M, Choo MS. Pretreatment features to influence effectiveness of intravesical hyaluronic acid instillation in refractory interstitial cystitis/painful bladder syndrome. Int Neurourol J. 2014; 18(3):163-167.

89. Hung MJ, Su TH, Lin YH, et al. Changes in sexual function of women with refractory interstitial cystitis/bladder pain syndrome after intravesical therapy with a hyaluronic acid solution. J Sex Med. 2014;11(9): 2256-2263.

90. Costantini E, Lazzeri M, Pistolesi D, et al. Morphological changes of bladder mucosa in patients who underwent instillation with combined sodium hyaluronic acid-chondroitin sulphate (Ialuril ${ }^{\circledR}$ ). Urol Int. 2013; 13:303.

91. Porru D, Leva F, Parmigiani A, et al. Impact of intravesical hyaluronic acid and chondroitin sulfate on bladder pain syndrome/interstitial cystitis. Int Urogynecol J. 2012;23:1193-1199.

92. Gülpınar O, Kayış A, Süer E, Gökçe Mİ, Güçlü AG, Arıkan N. Clinical comparision of intravesical hyaluronic acid and hyaluronic acidchondroitin sulphate therapy for patients with bladder pain syndrome/ interstitital cystitis. Can Urol Assoc J. 2014;8(9-10):E610-E614.

93. Laviana A, Jellison F, Kim JH. Sacral neuromodulation for refractory overactive bladder, interstitial cystitis, and painful bladder syndrome. Neurosurg Clin N Am. 2014;25(1):33-46.

94. Inkovic SP, Gillen LM, Marinkovic CM. Minimum 6-year outcomes for interstitial cystitis treated with sacral neuromodulation. Int Urogynecol J Pelvic Floor Dysfunct. 2011;22:407-412.

95. Gajewski JB, Al-Zahrani AA. The long-term efficacy of sacral neuromodulation in the management of in-tractable cases of bladder pain syndrome: 14 years of experience in one centre. BJU Int. 2011;107:1258-1264.

96. Fitzgerald MP, Anderson RU, Potts J, et al; Urological Pelvic Pain Collaborative Research Network. Randomized multicenter feasibility trial of myofascial physical therapy for the treatment of urological chronic pelvic pain syndromes. J Urol. 2013;189(1 supp1):S75-S85.

97. Lee ES, Lee SW, Lee KW, Kim JM, Kim YH, Kim ME. Effect of transurethral resection with hydrodistention for the treatment of ulcerative interstitial cystitis. Korean J Urol. 2013;54(10):682-688.

98. Peeker R, Aldenborg F, Fall M. Complete transurethral resection of ulcers inclassic interstitial cystitis. Int Urogynecol J Pelvic Floor Dysfunct. 2000;11:290.

99. Rofeim O, Hom D, Freid RM, Moldwin RM. Use of the neodymium: YAG laser forinterstitial cystitis: a prospective study. J Urol. 2001; 166:134-136.

100. Chennamsetty A, KhourdajiI, Goike J, KillingerKA, Girdler B, Peters KM. Electrosurgical management of hunner ulcers in a referral center's interstitial cystitis population. Urology. 2014;85(1):74-78.

101. Chong JT, Dolat MT, Klausner AP, Dragoescu E, Hampton LJ. The role of cystectomy for non-malignant bladder conditions: a review. Can J Urol. 2014;21(5):7433-7441.
102. Rössberger J, Fall M, Jonsson O, Peeker R. Long-term results of reconstructivesurgery in patients with bladder pain syndrome/ interstitial cystitis: subtypingis imperative. Urology. 2007;70: 638-642.

103. Hanno P. Editorial comment to efficacy and safety of augmentation ileocystoplasty combined with supratrigonalcystectomy for the treatment of refractory bladder pain syndrome/interstitial cystitis with Hunner's lesion. Int J Urol. 2014;21(suppl 1):74.

104. Chen H, Wang F, Chen W, et al. Efficacy of daily low-dose sildenafil for treating interstitial cystitis: results of a randomized, double-blind, placebo-controlled trial - treatment of interstitial cystitis/painful bladder syndrome with low-dose sildenafil. Urology. 2014;84(1):51-56.

105. Wang ZY, Wang P, Bjorling DE. Treatment with a cannabinoid receptor 2 agonist decreases severity of established cystitis. J Urol. 2014;191(4):1153-1158

106. Tambaro S, Casu MA, Mastinu A, Lazzari P. Evaluation of selective cannabinoid $\mathrm{CB}(1)$ and $\mathrm{CB}(2)$ receptor agonists in a mouse model of lipopolysaccharide-induced interstitial cystitis. Eur J Pharmacol. 2014;729:67-74.

107. Yokoyama H, Oguchi T, Goins WF, et al. Effects of herpes simplex virus vector-mediated enkephalin gene therapy on bladder overactivity and nociception. Hum Gene Ther. 2013;24(2):170-180.

108. Bosch PC. Examination of the significant placebo effect in the treatment of interstitial cystitis/bladder pain syndrome. Urology. 2014;84:321-326.

109. Lai HH. Clinical trials: placebo effects in interstitial cystitis/bladder pain syndrome. Nat Rev Urol. 2014;11:494-495.

110. Benedetti F. Drugs and placebos: what's the difference?: understanding the molecular basis of the placebo effect could help clinicians to better use it in clinical practice. EMBO Rep. 2014;15(4):329-332.

111. Buffington CAT, Westropp JL, Chew DJ. From FUS to Pandora syndrome: where are we, how did we get here, and where to now? J Feline Med Surg. 2014;16:385.

112. Valentino RJ, Van Bockstaele E. Convergent regulation of locus coeruleus activity as an adaptive response to stress. Eur J Pharmacol. 2008;583:194-203.

113. Reche AJ, Buffington CAT. Increased tyrosine hydroxylase immunoreactivity in the locus coeruleus of cats with interstitial cystitis. J Urol. 1998;159:1045-1048.

114. Charrua A, Pinto R, Taylor A, et al. Can the adrenergic system be implicated in the pathophysiology of bladder pain syndrome/interstitial cystitis? A clinical and experimental study. Neurourol Urodyn. 2013.

115. Yehuda R, Flory JD, Bierer LM, et al. Lower methylation of glucocorticoid receptor gene promoter $1 \mathrm{f}$ in peripheral blood of veterans with posttraumatic stress disorder. Biol Psychiatry. 2014;77(4): 356-364.

116. Castren E. Neuronal network plasticity and recovery from depression. JAMA Psychiatry. 2013;70:983-989.
International Journal of Women's Health

\section{Publish your work in this journal}

The International Journal of Women's Health is an international, peerreviewed open-access journal publishing original research, reports, editorials, reviews and commentaries on all aspects of women's healthcare including gynecology, obstetrics, and breast cancer. The manuscript management system is completely online and includes
Dovepress

a very quick and fair peer-review system, which is all easy to use. Visit http://www.dovepress.com/testimonials.php to read real quotes from published authors. 